\title{
A prospective observational study on trajectories and prognostic factors of mid back pain
}

\author{
Christina Knecht ${ }^{1}$, Sonja Hartnack ${ }^{2}$, Beate Sick ${ }^{3}$, Fabienne Riner ${ }^{1}$, Petra Schweinhardt ${ }^{1}$ and Brigitte Wirth ${ }^{*}$
}

\begin{abstract}
Background: Although mid back pain (MBP) is a common condition that causes significant disability, it has received little attention in research and knowledge about trajectories and prognosis of MBP is limited. The purpose of this study was to identify trajectories of MBP and baseline risk factors for an unfavorable outcome in MBP patients undergoing chiropractic treatment.

Methods: This prospective-observational study analyzes outcome data of 90 adult MBP patients (mean age $=$ $37.0 \pm 14.6$ years; 49 females) during one year (at baseline, after 1 week, 1 month, 3, 6 and 12 months) after start of chiropractic treatment. Patients completed an 11-point (0 to 10) numeric pain rating scale (NRS) at baseline and one week, one month, three, six and twelve months after treatment start and the Patient's Global Impression of Change $(\mathrm{PGIC})$ questionnaire at all time points except baseline. To determine trajectories, clustering with the package $\mathrm{kml}$ (software R), a variant of k-means clustering adapted for longitudinal data, was performed using the NRS-data. The identified NRS-clusters and PGIC data after three months were tested for association with baseline variables using univariable logistic regression analyses, conditional inference trees and random forest plots.
\end{abstract}

Results: Two distinct NRS-clusters indicating a favourable (rapid improvement within one month from moderate pain to persistent minor pain or recovery after one year, $80 \%$ of patients) and an unfavourable trajectory (persistent moderate to severe pain, $20 \%$ of patients) were identified. Chronic (> 3 months) pain duration at baseline significantly predicted that a patient was less likely to follow a favourable trajectory $[\mathrm{OR}=0.16,95 \% \mathrm{Cl}=0.05-0.50$, $p=0.002]$ and to report subjective improvement after twelve months [OR $=0.19,95 \% \mathrm{Cl}=0.07-0.51, p=0.001]$, which was confirmed by the conditional inference tree and the random forest analyses.

Conclusions: This prospective exploratory study identified two distinct MBP trajectories, representing a favourable and an unfavourable outcome over the course of one year after chiropractic treatment. Pain chronicity was the factor that influenced outcome measures using NRS or PGIC.

Keywords: Chiropractic, Cluster, Improvement, Mid back, Outcome, Pain, Risk factor, Spine, Thoracic, Trajectory

\footnotetext{
* Correspondence: brigitte.wirth@balgrist.ch

${ }^{1}$ Integrative Spinal Research, Department of Chiropractic Medicine, University

Hospital Balgrist, Forchstr. 340, 8008 Zurich, Switzerland

Full list of author information is available at the end of the article
}

(C) The Author(s). 2020 Open Access This article is licensed under a Creative Commons Attribution 4.0 International License, which permits use, sharing, adaptation, distribution and reproduction in any medium or format, as long as you give appropriate credit to the original author(s) and the source, provide a link to the Creative Commons licence, and indicate if changes were made. The images or other third party material in this article are included in the article's Creative Commons licence, unless indicated otherwise in a credit line to the material. If material is not included in the article's Creative Commons licence and your intended use is not permitted by statutory regulation or exceeds the permitted use, you will need to obtain permission directly from the copyright holder. To view a copy of this licence, visit http://creativecommons.org/licenses/by/4.0/ The Creative Commons Public Domain Dedication waiver (http://creativecommons.org/publicdomain/zero/1.0/) applies to the data made available in this article, unless otherwise stated in a credit line to the data. 


\section{Background}

Mid back pain (MBP) or thoracic spine pain, described as pain between the 1st and 12th thoracic vertebrae and the corresponding posterior aspect of the trunk [1] is a common condition in the general adult population (12-month prevalence rate $15-35 \%$ in the general adult population; median in most occupational groups around 30\% [2, 3]). Compared to low back pain (LBP) (12-month prevalence rates: 1-83\%; median 12-month prevalence rate: $37 \%$ [4]) and neck pain (NP) (12-month prevalence rates: $17-75 \%$; mean 12-months prevalence rate: 37\% [5]), MBP might be slightly less common in the adult population, although it can be equally disabling [3] and result in the same consequences [6, 7]. Nevertheless MBP has received considerably less attention than LBP and NP in research [2] and the thoracic spine has been called the 'Cinderella region' or step child of spinal research $[8,9]$.

A recent systematic review by Johansson and colleagues identified a knowledge gap with respect to MBP recovery trajectories and prognostic factors [10]. Because pain characteristics of the three spinal regions are similar $[6,7]$, suggesting that spinal pain might be a general disorder, the authors hypothesized that MBP trajectories might be comparable to those of NP and LBP [10]. Regarding prognostic factors, a systematic review on MBP identified only two prospective studies [3] and both focused on MBP in adolescence $[11,12]$. In adults, a more recent study reported poor patient expectations for recovery to be a prognostic factor for delayed recovery from traumatic MBP [13], while female gender and other concurrent musculoskeletal symptoms were crosssectionally associated with MBP [3].

To help closing the identified knowledge gaps in MBP research, the aim of this prospective observational study was to identify trajectories and risk factors for an unfavorable outcome in MBP patients undergoing chiropractic treatment.

\section{Methods}

\section{Participants and study design}

The present study analyzes data collected between June 2014 and December 2019 of an ongoing study tracking patients of a chiropractic teaching clinic affiliated with a mainly orthopedic university hospital in Switzerland. Patients over 18 years without any contra-indications to chiropractic manipulative treatment (e.g. tumors, infections, inflammatory spondylarthropathies, acute fractures and severe osteoporosis) completed an 11-point (0 to 10) numeric rating scale (NRS) for present pain intensity before the first chiropractic treatment (baseline) and after one week, one month and after three, six and twelve months. In addition, they completed the Patient's Global Impression of Change (PGIC), a seven point Likert-scale assessing the patient's rating of overall improvement with the extremes "much worse" and "much better" [14] at all time points apart from baseline. Lastly, data on pain duration, previous MBP episodes, presence of concurrent LBP or NP, pain onset (traumatic or non-traumatic), smoking habits and rib involvement (based on the chiropractor's diagnosis) was assessed. After written informed consent was obtained, the questionnaires were administered to the patients by the treating chiropractor immediately before the first treatment. Patients chose whether they preferred to answer the follow-up questionnaires via email or phone. If phone contact was preferred, a trained research assistant who did not know the patient conducted telephone interviews at each time point, irrespective of whether the patient was still undergoing chiropractic treatment or not. If online contact was chosen, survey invitations were sent to the participants using the software REDCap (version 8.3.2), a secure web-based application designed to support data capture for research studies [15]. This study was approved by the Ethics review board of the Canton of Zurich (EK-16/2009; update PB_2017-00402).

\section{Data analysis and statistics}

Patients for whom NRS data were available from at least five out of the six time points were included. To handle missing data, the random forest package (MissForest [16]), a non-parametric imputation method for mixed-type data, was used. This method uses a random forest trained on the observed data to predict the missing values without the need for a test set or cross validation. In the present study, $4.6 \%$ of all values were missing with a maximum of 12 missing values $(=13.3 \%)$ in the variable 'PGIC after 1 week'.

Repeated measures ANOVA was conducted to test for the significance of NRS changes between baseline and one year after treatment start. For the PGIC, the two highest categories ("much better" and "better") were defined as clinically relevant improvement [17, 18]. The McNemar test was used to test whether the percentage of patients who reported improvement significantly changed over time. Descriptive statistical analyses were conducted using the non-imputed data. All inferential analyses used the imputed data set.

Clustering with the package $\mathrm{KmL}$ (statistical software $\mathrm{R}$ 3.4.2; Boston, MA), a variant of $\mathrm{k}$-means clustering specifically designed for longitudinal data, was performed to determine trajectories $[19,20]$. The algorithm reaches the optimal cluster number by alternating an 'expectation phase', where the center of each cluster is determined, and a 'maximization phase' that assigns each observation to its nearest cluster. This alternation is repeated until no further changes in the clusters occur. To determine the optimal number of clusters, five criteria were used to strengthen the reliability of the result (Calinski \& Harabatz, Ray \& Turi, Davies \& Bouldin). These criteria assess the quality of 
partition by combining the between-cluster covariance and the within-cluster covariance $[19,20]$.

The obtained clusters were subsequently tested for association with baseline factors using univariable logistic regression analysis with cluster membership as the dependent variable. The independent variables were age, gender, pain duration (acute/subacute versus chronic; acute and subacute patients were summarized into one category because medium (1 month) and long-term ( $\geq 3$ months) outcome was shown to be comparable for these groups in NP [21]), previous episodes (no previous episode versus $\geq 1$ previous episodes), other painful areas (no concurrent versus concurrent LBP or NP or headache), whether the treating chiropractor diagnosed rib involvement (costo-vertebral syndrome yes/no), traumatic onset (yes/no), and smoking status (yes/no). To test the effects of these baseline factors on the patients' perception of outcome (PGIC) after twelve months, univariable binary logistic regression models with the dependent variable PGIC (dichotomized; 'improved' = better or much better, rest = 'not improved') were run. The univariable logistic regression approach was chosen because the statistical power was too low to include all these potential risk factors in the same multivariable regression model and the approach of using univariable analyses as an initial step to select covariates for further consideration in a multivariable model was shown to be invalid as this might lead to biased estimates [22]. Instead the multivariable model was investigated using conditional inference trees [23-25] and random forest plots [26]. Tree-based methods are an alternative to logistic regression to reveal risk factors [27] and are increasingly used in clinical problems [28]. To test for multicollinearity, pairwise associations were tested with Fisher's exact tests.

Descriptive statistics, repeated measures ANOVA and logistic regression analyses were performed using SPSS 24.0 (IBM Corp., Chicago, IL). Data imputation, determination of trajectories, the conditional inference trees and random forest plots were performed using the statistical software R (statistical software R 3.4.2; Boston, MA [29]). For all analyses, a $p$-value of $<0.05$ was considered statistically significant.

\section{Results}

Ninety patients (mean age $=37.0 \pm 14.6$ years) of whom $49(54.4 \%)$ were female were included in the analysis $(84$ patients were excluded due to fewer than five data sets). Of these, 15 patients chose to be contacted via phone and 75 patients answered the follow-up questionnaires using the provided electronic link. For the majority of patients $(N=57 / 64.8 \%)$, the current MBP episode was the first, while 11 patients (12.5\%) had suffered from one to three previous episodes and 20 patients (22.7\%) from more than three episodes (2 missing values). Fourtyeight $(53.9 \%)$ patients were acute (pain duration $\leq 30$ days), 8 (9.0\%) subacute patients (pain duration 31-90 days) and 33 (37.1\%) chronic patients (pain duration > 90 days) (1 missing value). Thirty-four patients (37.8\%) reported concurrent LBP, NP or LBP and NP. Ten patients (11.4\%) reported MBP of traumatic origin (2 missing values), and rib involvement was reported by the treating chiropractor in 36 patients $(40.0 \%)$. Of the 52 patients without rib involvement, 25 (27.8\%) were diagnosed with axial pain and $29(32.2 \%)$ were diagnosed with somatic referred pain. Twenty-four patients (27.0\%) were smokers ( 1 missing value). These patients were comparable to those who were excluded from the study because they did not provide data of at least five of the six time points and did not statistically differ in any of the variables mentioned above.

NRS values significantly improved within the first twelve months after start of chiropractic treatment from $5.03( \pm$ SD 2.06) points at baseline to 1.81 ( \pm SD 2.21) points $\left[\mathrm{F}_{(192.93,3.56)}=54.16, p<0.001\right]$. Post-hoc tests showed significant pain reduction from baseline to all time points (p's $<0.001$ ) and from one week to all time points (p's $<$ 0.001). Afterwards, no significant pain reduction occurred up to one year after treatment start (Fig. 1).

The percentage of patients reporting improvement in the PGIC significantly increased from $N=38 / 48.7 \%$ after one week (12 missing values) to $N=58 / 70.7 \%$ after one month $(8$ missing values) $(p<0.001)$, where it stabilized [improvement after three months $N=60 / 69.8 \%, 4$ missing values; after six and twelve months $\mathrm{N}=60 / 72.3 \%, 7$ missing values]. Using the imputed data set, the results were comparable (improvement $=45.6 \%$ after 1 week, $70.0 \%$ after 1 month, 71.1\% after 3, 6 and 12 months).

\section{Trajectories}

The five criteria indicated highest partition quality for a two- and six-cluster solution: three criteria were in favor of two clusters and two criteria in favor of six clusters (Fig. 2).

Using the terminology by Kongsted and colleagues for LBP trajectories [30], the trajectory 1 ('improvers') in the two-cluster solution describes rapid improvement within one month from moderate pain to persistent minor pain or recovery [30] after one year. The trajectory 2 ('nonimprovers') describes persistent moderate to severe pain [30]. The majority of patients $(N=72 / 80 \%)$ followed trajectory $1(N=51 / 70.8 \%$ were acute/subacute), and 18 patients $(20 \%)$ followed trajectory $2(\mathrm{~N}=5 / 27.8 \%$ were acute/subacute). Thus, $91.1 \%$ from the acute/subacute patients and $61.8 \%$ from the chronic patients followed trajectory 1 (Fig. 3). Of note, these numbers can slightly vary when choosing different seeds in the model due to the stochastic nature of the analysis.

In the six-cluster solution selected by two criteria with the same high partition quality as the two-cluster solution, four clusters, starting either from mild (Cluster C), 

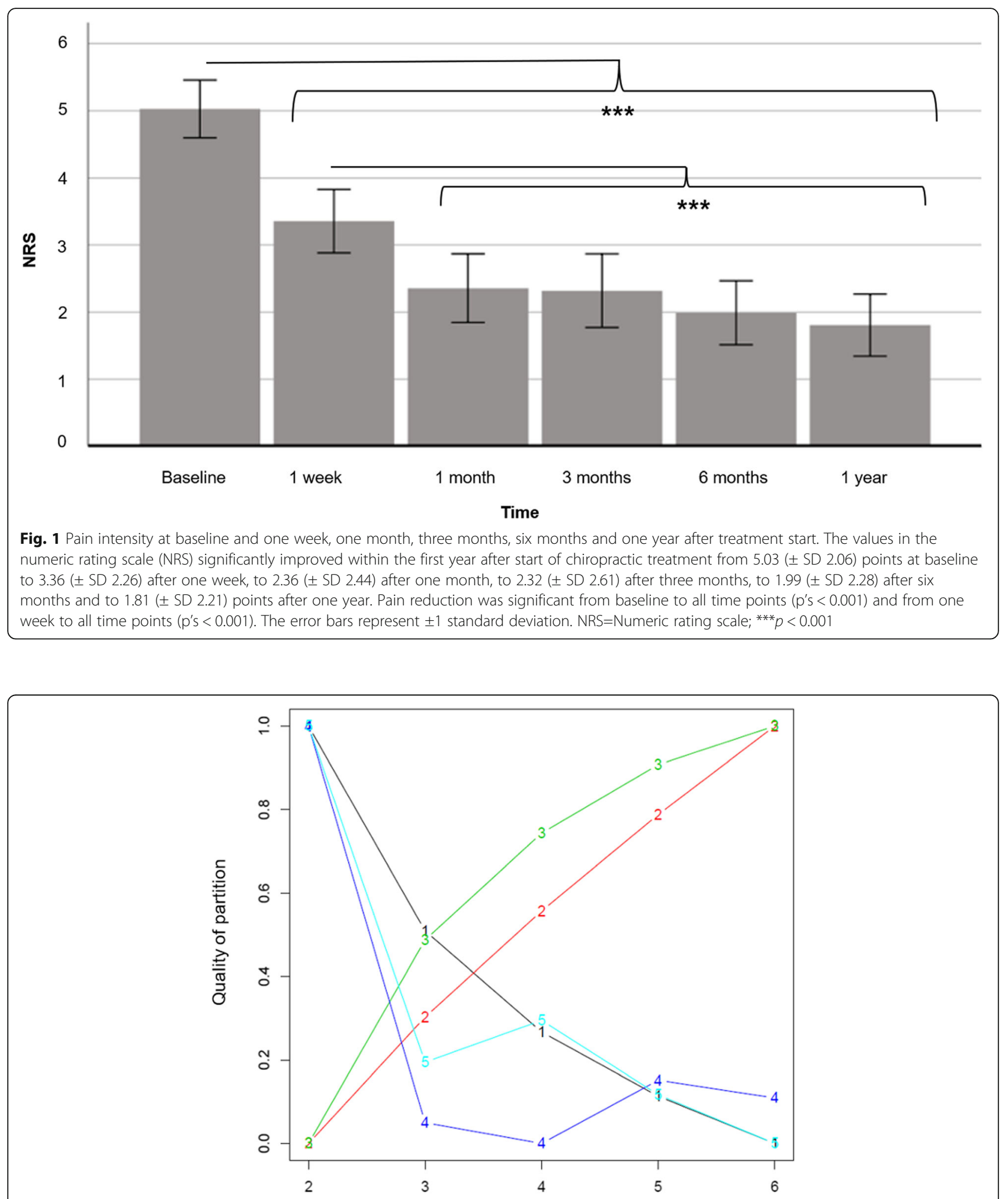

Number of clusters

Fig. 2 Partition quality of cluster analysis. The $x$-axis shows the number of clusters, the $y$-axis the quality of the partition, which represents a combination of the within- and the between-cluster covariance. A high value indicates good partition quality. A two-cluster solution was favored by three criteria ( $1=$ Calinski Harabatz 1; $4=$ Ray Turi; $5=$ Davies Bouldin), a six-cluster solution by two criteria $(2=$ Calinski Harabatz 2; $3=$ Calinski Harabatz 3) 


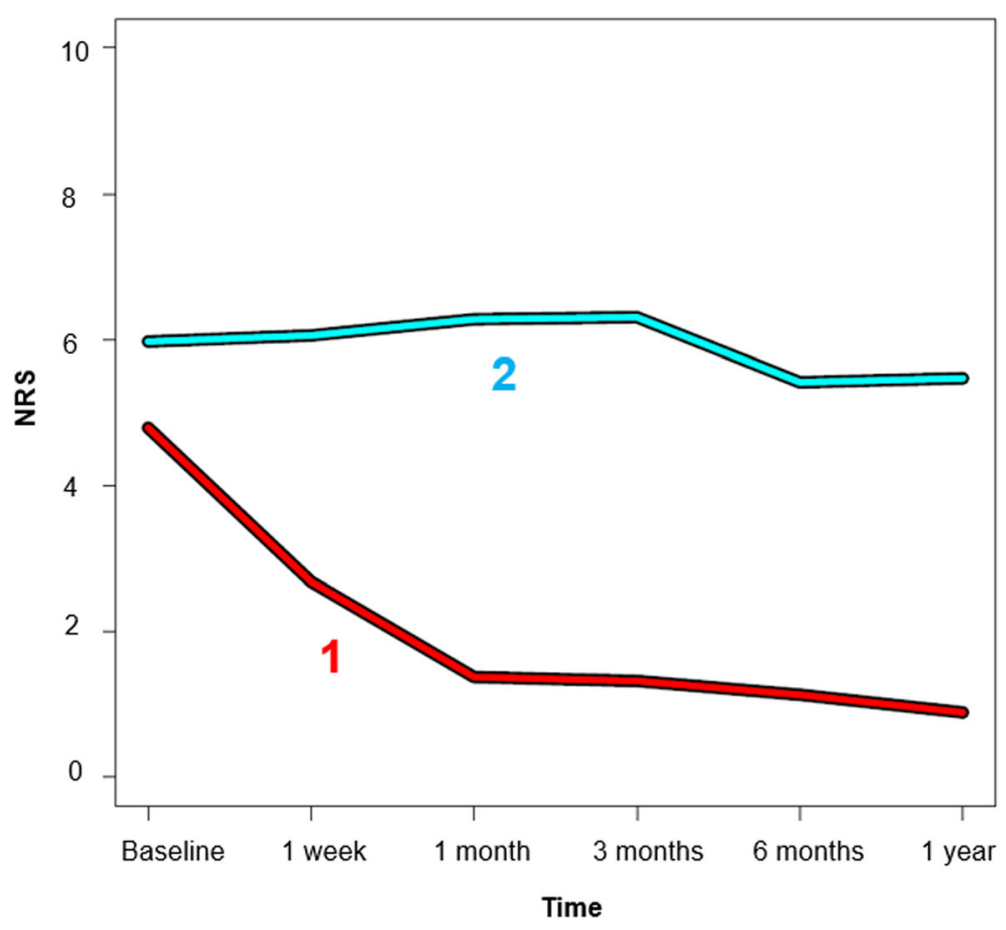

Fig. 3 Two-cluster solution. Trajectory 1 (red) represents the 'improvers' (rapid recovery from moderate pain and persistent minor pain), trajectory 2 (blue) represents the 'non-improvers' (persistent moderate pain)

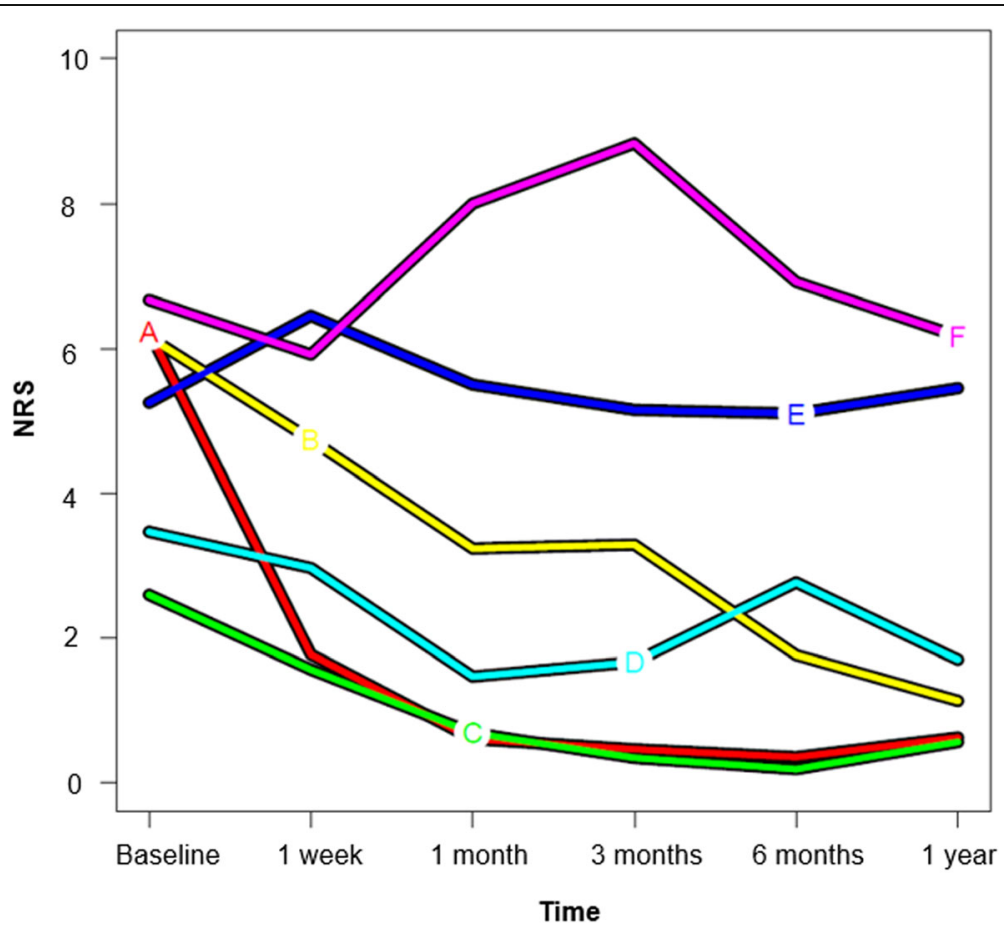

Fig. 4 Six-cluster solution. The trajectories describe rapidly improving pain, starting either from mild (Cluster C, green), moderate (Cluster D, light blue) or from severe pain (Cluster A, red; Cluster B, yellow) describe rapidly improving pain ('marked decrease in pain intensity within one month' [30]) followed by recovery/minor pain (Cluster A and C) or mild pain (Cluster B and D) [30] after one year with one cluster (Cluster D) showing some slight pain exacerbation at three months 
moderate (Cluster D) or from severe pain (Cluster A and B) describe rapidly improving pain ('marked decrease in pain intensity within one month' [30]) followed by recovery/minor pain (Cluster $\mathrm{A}$ and $\mathrm{C}$ ) or mild pain (Cluster B and D) [30] after one year with one cluster (Cluster D) showing some slight pain exacerbation at six months (Fig. 4). Of the acute patients, 91-95\% followed one of these trajectories, while $59-65 \%$ of the chronic patients showed one of these pain patterns. On the higher end of the NRS-scale, two clusters (Cluster E and F) describe persistent moderate to severe pain [30]) after 12 months, either after prior gradual improvement or some temporary exacerbation. These pain patterns were followed by $5-9 \%$ of the acute and $35-41 \%$ of the chronic patients.

\section{Predictive factors}

Traumatic onset (Nagelkerke $\mathrm{R}^{2}=0.09$ ) and chronic pain (Nagelkerke $R^{2}=0.18$ ) were the factors that predicted the unfavorable trajectory (trajectory 2 ) in the univariable logistic regression models based on NRS data (Table 1). The factors age, gender, previous pain episodes, concurrent pain areas, rib involvement, and smoking status were not found to influence the trajectory.

In the conditional inference tree analysis using the same factors as the univariable models as independent variables, chronic pain was the only significant predictor for becoming a 'non-improver' $(p=0.006)$, which was confirmed as being the most important factor (highest mean decrease accuracy) in the random forest analysis (estimate of error rate $20.0 \%$ ). Similarly, pain duration longer than 3 months predicted no improvement in the PGIC 12 months after treatment start in the univariable logistic regression models (Nagelkerke $R^{2}=0.17$ ) (Table 2), and this was confirmed in the conditional inference tree analysis $(p=$ 0.002 ) and in the random forest analysis (estimate of error rate $34.44 \%$ ). The Fisher's exact tests for testing pairwise associations found only one significant association, namely between the factors 'other complaints' and 'rib involvement' $(p=0.009)$ indicating that multicollinearity was a minor problem in the model.

\section{Discussion}

In this population of mostly acute and subacute MBP patients undergoing chiropractic treatment, two trajectories emerged as the optimal solution of the cluster analysis, namely the 'improvers' (rapid recovery within one month and full recovery or persistent mild pain, $62 \%$ ) and the 'non-improvers' (persistent moderate pain, 38\%). The second best partition was a six-cluster solution revealing a more fine grained representation of trajectories. Pain longer than three months before treatment start significantly predicted that a patient followed the 'non-improver' trajectory in the two-cluster solution and reported nonimprovement in the PGIC after three months.

\section{Trajectories}

Because of the lack of information in the literature regarding MBP trajectories and prognostic factors [10], the trajectories observed in this study cannot be compared to other MBP studies. However, it has been hypothesized that MBP trajectories might be similar to those of LBP as pain characteristics of the three spinal regions, including NP, are comparable, suggesting that spinal pain is the general underlying disorder $[6,7]$.

In acute LBP patients, Downie and colleagues [31] and Kongsted and colleagues [32] found a majority of patients recovering (70\% [31] and 60\% [32], respectively), either rapidly or gradually, and a minority of patients with persistent severe pain (5\% [31] and 3\% [32], respectively). Similarly, a smaller study in 108 patients with a first episode of acute LBP reported recovery in $67 \%$ and persistent severe pain in $7 \%$ of patients [33]. These findings are compatible with the two-cluster solution (9\% of acute MBP patients with persistent severe pain) in the present study. In chronic LBP patients, Tamcan and colleagues depicted the course of untreated LBP in the general population and did not observe any improvement (defined as 'marked decrease in pain intensity' [30]) [34]. Macedo and colleagues analyzed trajectories over one year in 155 chronic LBP patients of all arms of an RCT on the effects of motor control exercises versus a graded activity intervention using cluster

Table 1 Predictors for trajectory. Results of univariable binary logistic models

\begin{tabular}{|c|c|c|}
\hline Trajectory 1 ('improvers', 1) versus trajectory 2 ('non-improvers', 0) & OR $(95 \% \mathrm{Cl})$ & $\boldsymbol{p}$-value \\
\hline Age (years) & $1.00(0.97-1.04)$ & 0.836 \\
\hline Gender male (0), female (1) & $0.53(0.18-1.56)$ & 0.249 \\
\hline Pain duration acute/subacute $\leq 3$ months (0), chronic $>3$ months (1) & $0.16(0.05-0.50)$ & 0.002 \\
\hline Previous episodes no previous episode (0), $\geq 1$ previous episodes (1) & $1.56(0.50-4.86)$ & 0.443 \\
\hline Other areas of complaint none or other complaints than LBP or neck pain (0), LBP and/or neck pain (1) & $0.60(0.21-1.70)$ & 0.336 \\
\hline Traumatic onset no (0), yes (1) & $0.19(0.05-0.77)$ & 0.019 \\
\hline Rib involvement no (0), yes (1) & $1.19(0.41-3.42)$ & 0.749 \\
\hline Smoker no $(0)$, yes $(1)$ & $0.93(0.29-2.96)$ & 0.905 \\
\hline
\end{tabular}

OR Odds Ratio, CI Confidence Interval, LBP Low Back Pain. Significant results are marked in bold. 
Table 2 Predictors for Patient's Global Impression of Change (PGIC) three months after treatment start. Results of univariable binary logistic models

\begin{tabular}{|c|c|c|}
\hline PGIC after 3 months not improved (0) vs. improved (1) & OR $(95 \% \mathrm{Cl})$ & $p$-value \\
\hline Age (years) & $1.01(0.97-1.04)$ & 0.747 \\
\hline Gender male (0), female (1) & $0.83(0.33-2.09)$ & 0.693 \\
\hline Pain duration acute/subacute $\leq 3$ months (0), chronic $>3$ months (1) & $0.19(0.07-0.51)$ & 0.001 \\
\hline Previous episodes no previous episode (0), $\geq 1$ previous episodes (1) & $2.28(0.81-6.45)$ & 0.120 \\
\hline Other areas of complaint none or other complaints than LBP or neck pain (0), LBP and/or neck pain (1) & $0.87(0.35-2.21)$ & 0.776 \\
\hline Traumatic onset no (0), yes (1) & $0.57(0.15-2.21)$ & 0.415 \\
\hline Rib involvement no (0), yes (1) & $1.24(0.49-3.16)$ & 0.645 \\
\hline Smoker no (0), yes (1) & $0.75(0.27-2.05)$ & 0.575 \\
\hline
\end{tabular}

OR Odds Ratio, CI Confidence Interval, LBP Low Back Pain. Significant results are marked in bold.

analysis [35]. They identified three clusters, which were gradual improvement ('marked decrease in pain intensity occurring gradually over more than one month' [30]) with persistent mild pain in $47 \%$, persistent moderate pain in $25 \%$ and persistent high pain in $15 \%$ of the patients. These clusters compare well with the clusters A-D (persistent minor or mild pain) and the clusters $\mathrm{E}$ and $\mathrm{F}$ (persistent moderate or high pain) in the present study. In summary, the MBP trajectories observed here are similar to those described for LBP. Acute spinal pain mostly follows a recovery trajectory with a minority of patients developing persistent or fluctuating pain. In contrast, chronic spinal pain recovers in a minority of patients and persists on a mild, moderate or severe pain level in many instances.

\section{Factors predictive for poor outcome}

Pain duration longer than three months before treatment start was associated with poor outcome in the present study reflected in the patients following an unfavorable trajectory based on NRS data and in the patients reporting no overall improvement in the PGIC, although this association was relatively low, with pain duration explaining $18 \%$ (NRS) and 17\% (PGIC) of the variance in outcome $\left(R^{2}=0.18\right.$ and 0.17$)$. In accordance, MBP of longer duration ( $>30$ days) was associated with a higher incidence rate for sickness absence than acute MBP [10]. Overall, the reported improvement was comparable to that of LBP and NP $[18,36]$ and the finding of higher percentages of self-reported overall improvement in acute compared to chronic MBP supports previous findings in LBP and NP $[18,36]$. Altogether, these findings confirm that longer pain duration is a risk factor for poor outcome of musculoskeletal pain as shown in a systematic review [37].

\section{Limitations}

Because the present study design did not include a control group, these data do not allow for drawing any conclusions about the effectiveness of chiropractic treatment. Instead, the data might depict the natural history of MPB. Furthermore, the relatively small number of MPB patients, their heterogeneity with respect to symptom duration and the fact that the MBP patients were recruited from a teaching clinic at a university hospital might limit the generalizability of the present study. Lastly, several factors possibly influencing the course and outcome of MBP, such as other concurrent therapies, medication, pre-existing comorbidities and physical activity were not included in the analysis. With regard to imaging it should be noted that approximately $90 \%$ of back problems are non-specific without any pathoanatomical cause that would be identifiable using imaging [38]. Therefore, guidelines discourage from routine imaging in these patients [39].

\section{Conclusion}

This study is a first step towards identifying trajectories in MBP, which were shown to be comparable to those of LBP. Pain duration longer than three months emerged as main risk factor for poor outcome. These findings support the notion that LBP and MBP belong to the same general disorder, which needs being confirmed in larger studies in the future.

\section{Abbreviations \\ LBP: Low back pain; MBP: Mid back pain; NP: Neck pain; NRS: Numeric pain rating scale; PGIC: Patient's Global Impression of Change; SD: Standard deviation}

Acknowledgements

We thank all patients for study participation.

\section{Authors' contributions}

CK contributed to the conception of the study, did parts of the statistical analysis, was involved in data interpretation and drafted the article. SH was involved in the conception of the study, did the main part of the statistical analysis and contributed to data interpretation. BS was involved in the conception of the statistical analysis and in data interpretation. FR did the main part of data acquisition and was involved in writing the manuscript. PSch contributed to the conception of the study, was involved in data interpretation and in writing the manuscript. BW contributed to the conception of the study, was involved in data acquisition and data interpretation and wrote the final manuscript. All authors contributed to and approved the final manuscript.

\section{Funding}

BW receives salary support from the Swiss Chiropractors' Association Chirosuisse. 


\section{Availability of data and materials}

The datasets used are available from the corresponding author upon request.

\section{Ethical approval and consent to participate}

This study was approved by the Ethics review board of the Canton of Zurich (EK-16/ 2009; update PB_2017-00402) and all participants gave informed written consent.

\section{Consent for publication}

Not applicable.

\section{Competing interests}

The authors declare no competing interests.

\section{Author details}

'Integrative Spinal Research, Department of Chiropractic Medicine, University Hospital Balgrist, Forchstr. 340, 8008 Zurich, Switzerland. ${ }^{2}$ Section of Epidemiology, Vetsuisse Faculty, University of Zurich, Winterthurerstr. 270, 8057 Zurich, Switzerland. ${ }^{3}$ University of Zurich, Epidemiology, Biostatistics \& Prevention Institute (EBPI), Hirschengraben 84, 8001 Zurich, Switzerland.

Received: 18 March 2020 Accepted: 23 July 2020

Published online: 17 August 2020

\section{References}

1. Leboeuf-Yde C, Nielsen J, Kyvik KO, Fejer R, Hartvigsen J. Pain in the lumbar, thoracic or cervical regions: do age and gender matter? A population-based study of 34,902 Danish twins 20-71 years of age. BMC Musculoskelet Disord. 2009;10:39. https://doi.org/10.1186/1471-2474-10-39.

2. Briggs AM, Bragge P, Smith AJ, Govil D, Straker LM. Prevalence and associated factors for thoracic spine pain in the adult working population: a literature review. J Occup Health. 2009:51:177-92.

3. Briggs AM, Smith AJ, Straker LM, Bragge P. Thoracic spine pain in the general population: prevalence, incidence and associated factors in children, adolescents and adults. Syst Rev BMC Musculoskelet Disord. 2009;10:77. https://doi.org/10.1186/1471-2474-10-77.

4. Hoy D, Bain C, Williams G, March L, Brooks P, Blyth F, et al. A systematic review of the global prevalence of low back pain. Arthritis Rheum. 2012;64: 2028-37. https://doi.org/10.1002/art.34347.

5. Fejer R, Kyvik KO, Hartvigsen J. The prevalence of neck pain in the world population: a systematic critical review of the literature. Eur Spine J. 2006;15: 834-48. https://doi.org/10.1007/s00586-004-0864-4

6. Leboeuf-Yde C, Fejer R, Nielsen J, Kyvik KO, Hartvigsen J. Pain in the three spinal regions: the same disorder? Data from a population-based sample of 34,902 Danish adults. Chiropr Man Therap. 2012;20:11. https:/doi.org/10.1186/2045-709X-20-11.

7. Leboeuf-Yde C, Fejer R, Nielsen J, Kyvik KO, Hartvigsen J. Consequences of spinal pain: do age and gender matter? A Danish cross-sectional population-based study of 34,902 individuals 20-71 years of age. BMC Musculoskelet Disord. 2011:12:39. https://doi.org/10.1186/1471-2474-12-39.

8. Heneghan NR, Rushton A. Understanding why the thoracic region is the 'Cinderella' region of the spine. Man Ther. 2016;21:274-6. https:/doi.org/10.1016/.math.2015.06.010.

9. Haldeman S. Thoracic pain in adolescents: another untreatable disease that could consume resources and increase disability? Spine J. 2009;9:339-40. https://doi.org/10.1016/j.spinee.2009.01.002.

10. Johansson MS, Jensen Stochkendahl M, Hartvigsen J, Boyle E, Cassidy JD. Incidence and prognosis of mid-back pain in the general population: a systematic review. Eur J Pain. 2017;21:20-8. https://doi.org/10.1002/ejp.884.

11. Grimmer K, Nyland L, Milanese S. Repeated measures of recent headache, neck and upper back pain in Australian adolescents. Cephalalgia. 2006;26: 843-51. https://doi.org/10.1111/j.1468-2982.2006.01120.x.

12. Ehrmann Feldman D, Shrier I, Rossignol M, Abenhaim L. Risk factors for the development of neck and upper limb pain in adolescents. Spine. 2002;27:523-8.

13. Johansson MS, Boyle E, Hartvigsen J, Jensen Stochkendahl M, Carroll L, Cassidy JD. A population-based, incidence cohort study of mid-back pain after traffic collisions: factors associated with global recovery. Eur J Pain. 2015;19:1486-95. https://doi.org/10.1002/ejp.681.

14. Fischer D, Stewart AL, Bloch DA, Lorig K, Laurent D, Holman H. Capturing the patient's view of change as a clinical outcome measure. JAMA. 1999;282:1157-62.

15. Harris PA, Taylor R, Thielke R, Payne J, Gonzalez N, Conde JG. Research electronic data capture (REDCap)--a metadata-driven methodology and workflow process for providing translational research informatics support. J Biomed Inform. 2009;42:377-81. https://doi.org/10.1016/j.jbi.2008.08.010.
16. Stekhoven DJ, Bühlmann P. MissForest--non-parametric missing value imputation for mixed-type data. Bioinformatics. 2012;28:112-8. https://doi. org/10.1093/bioinformatics/btr597.

17. Hurst $\mathrm{H}$, Bolton J. Assessing the clinical significance of change scores recorded on subjective outcome measures. J Manip Physiol Ther. 2004;27: 26-35. https://doi.org/10.1016/j.jmpt.2003.11.003.

18. Peterson CK, Bolton J, Humphreys BK. Predictors of improvement in patients with acute and chronic low back pain undergoing chiropractic treatment. J Manip Physiol Ther. 2012;35:525-33. https://doi.org/10.1016/j.jmpt.2012.06.003.

19. Genolini C, Alacoque X, Sentenac M, Arnaud C. Kml and kml3d: R Packages to Cluster Longitudinal Data. J Stat Soft. 2015. https://doi.org/10.18637/jss.v065.04.

20. Genolini C, Falissard B. KmL: a package to cluster longitudinal data. Comput Methods Prog Biomed. 2011;104:e112-21. https://doi.org/10.1016/..cmpb.2011.05.008.

21. Nyirö L, Peterson CK, Humphreys BK. Exploring the definition of «acute» neck pain: a prospective cohort observational study comparing the outcomes of chiropractic patients with 0-2 weeks, 2-4 weeks and 4-12 weeks of symptoms. Chiropr Man Therap. 2017;25:24. https://doi.org/10.1186/s12998-017-0154-y.

22. Wang H, Peng J, Wang B, Lu X, Zheng JZ, Wang K, et al. Inconsistency Between Univariate and Multiple Logistic Regressions. Shanghai Arch Psychiatr. 2017;29:124-8. https://doi.org/10.11919/j.issn.1002-0829.217031.

23. Hothorn T, Zeileis A. Partykit: a modular toolkit for recursive partytioning in R. J Machine Learn Res. 2015:3905-9.

24. Hothorn T, Hornik K, Zeileis A. Unbiased recursive partitioning: a conditional inference framework. J Comput Graph Stat. 2006;15:651-74. https://doi.org/ 10.1198/106186006X133933.

25. Therneau T, Atkinson B. Package 'rpart'. 2019. https://cran.r-project.org/web/ packages/rpart/index.html. Accessed July 8th, 2019.

26. Liaw A, Wiener M. Classification and regression by random forest. R News. 2002;2002:18-22.

27. Nagy K, Reiczigel J, Harnos A, Schrott A, Kabai P. Tree-based methods as an alternative to logistic regression in revealing risk factors of crib-biting in horses. J Equine Vet Sci. 2010;30:21-6. https://doi.org/10.1016/j.jevs.2009.11.005.

28. Sanchez-Pinto LN, Venable LR, Fahrenbach J, Churpek MM. Comparison of variable selection methods for clinical predictive modeling. Int J Med Inform. 2018;116:10-7. https://doi.org/10.1016/j.jmedinf.2018.05.006.

29. R Core Team. R: A language and environment for statistical computing. 2014. https://www.R-project.org/.

30. Kongsted A, Kent P, Axen I, Downie AS, Dunn KM. What have we learned from ten years of trajectory research in low back pain? BMC Musculoskelet Disord. 2016;17:220. https://doi.org/10.1186/s12891-016-1071-2.

31. Downie AS, Hancock MJ, Rzewuska M, Williams CM, Lin C-WC, Maher CG. Trajectories of acute low back pain: a latent class growth analysis. Pain. 2016;157:225-34. https://doi.org/10.1097/j.pain.0000000000000351.

32. Kongsted $A$, Kent $P$, Hestbaek $L$, Vach W. Patients with low back pain had distinct clinical course patterns that were typically neither complete recovery nor constant pain. A latent class analysis of longitudinal data. Spine J. 2015;15:885-94. https://doi.org/10.1016/.jpinee.2015.02.012.

33. Wirth $B$, Ehrler M, Humphreys BK. First episode of acute low back pain - an exploratory cluster analysis approach for early detection of unfavorable recovery. Disabil Rehabil. 2017;39:2559-65. https:/doi.org/10.1080/09638288.2016.1239765.

34. Tamcan O, Mannion AF, Eisenring C, Horisberger B, Elfering A, Müller U. The course of chronic and recurrent low back pain in the general population. Pain. 2010;150:451-7. https://doi.org/10.1016/j.pain.2010.05.019.

35. Macedo LG, Maher CG, Latimer J, McAuley JH, Hodges PW, Rogers WT. Nature and determinants of the course of chronic low back pain over a 12-month period: a cluster analysis. Phys Ther. 2014;94:210-21. https:/doi.org/10.2522/ptj.20120416.

36. Peterson C, Bolton J, Humphreys BK. Predictors of outcome in neck pain patients undergoing chiropractic care: comparison of acute and chronic patients. Chiropr Man Therap. 2012;20:27. https://doi.org/10.1186/2045-709X-20-27.

37. Mallen CD, Peat G, Thomas E, Dunn KM, Croft PR. Prognostic factors for musculoskeletal pain in primary care: a systematic review. Br J Gen Pract 2007:57:655-61.

38. Oliveira C, Maher C, Pinto R, Traeger A, Lin C, Chenot J, van Tulder M, Koes B. Clinical practice guidelines for the management of non-specific low back pain in primary care: an updated overview. Eur Spine J. 2018;27:2791-803.

39. Maher $\mathrm{C}$, Underwood M, Buchbinder R. Non-specific low back pain. Lancet. 2017:389:736-47.

\section{Publisher's Note}

Springer Nature remains neutral with regard to jurisdictional claims in published maps and institutional affiliations. 